\title{
How To Make a Referral for Psychologic Testing Understood and Accepted
}

\author{
Some Suggestions for the Pediatrician
}

Edward M. Schwartz, Ph.D.*

W

HEN A PEDIATRICIAN requests psychologic testing of a patient, it is not uncommon for both child and parents to have anxiety about the referral, reflecting their own insufficient or distorted information and lack of understanding of the testing process. Consequently, the pediatrician should explore these concerns and discuss them with the family before they hamper the acceptance of the evaluation. In addition, pediatrician awareness of the child and parent reactions makes the collaboration between himself and the psychologist more effective, and avoids having the family puzzled by superficially conflicting views and explanations among the professionals.

Perhaps the greatest concern of parents when told of the need for psychologic testing is that they will not appropriately prepare the child, and therefore his test responses may not be truly representative. They fear that if their child "fails" the test he will be forever stigmatized by the "poor" performance. This is a particularly relevant worry of parents who are facing the prospect that their child is mentally retarded or learning disabled.

Parents, therefore, should be made to understand in advance that the testing is not a pass-fail situation nor will the child's per-

* Assistant Professor of Psychology, Department of Pediatrics and Communicable Diseases, The University of Michigan, School of Medicine, Ann Arbor, Mich. 48104 . formance be summarized in a single score. A description of the testing process is in order, pointing out that there will be a variety of demands, problems, and stimuli of which no child can be expected to be completely familiar. Since the child's learning problem is under study, the evaluation must provide a sample of his usual responses in a learning situation. Preparation, therefore, is actually inappropriate for the testing. When explained as such to the parents, this information ordinarily relieves their worry that they should do something to prepare the child.

Many parents fear that their child may not cooperate with the examiner during the evaluation. Here, again, one encounters a preconceived and erroneous conception that the psychologic tests are designed primarily to add up right-wrong responses and arrive at a tally. These parents should be assured that an uncooperative (or fearful, aggressive, or hyperactive) child is not a problem in the testing if that behavior at least partly reflects the child's typical response pattern in the learning situation and/ or in getting along with others. That a child is uncooperative may become a major focus of the evaluation in order to explore both the motivation for the behavior and appropriate ways of intervening with the child. The parents can be assured that the psychologist is a specialist in working with and understanding children and will view 


\section{SCHWARTZ}

test responses within the context of the child's feelings, behavior, and reactions to the test situation.

Unlike when parents tell a child he is going to a doctor for a checkup or some treatment for an illness, explaining an appointment for psychologic testing is a different matter. That the child should receive a factual explanation in advance has become evident from the many children we see who are in a most anxious state on arrival because of fantasies and gross distortions about what is wrong with them. We recommend strongly that the pediatrician when advising a referral explain the subject directly and honestly with the child, keeping in mind of course the child's developmental level and capacity for understanding. Basically, the child should be told simply that because of poor learning in school, getting along with parents or peers, feeling worried much of the time, etc., both the parents and the pediatrician are concerned and that the child is going to see a person who may be of help with these problems, i.e., a psychologist. Most children will readily understand and accept this explanation. Contrariwise, when told that he is going for "another checkup" or to "see a friend of the doctor's" that a child grows suspicious and anxious. In addition, the pediatrician should encourage the parents to discuss the evaluation directly with the child. This approach may become the first step toward helping both child and parents deal with the problem for which the evaluation has been requested.

Even in this age of enlightenment, many persons feel a social stigma in seeking a mental health professional for evaluation, guidance, or counseling. Reassurance about the confidentiality of the results is the most effective way for the pediatrician to minimize such concern.

The request for psychologic evaluation of a child with psychosomatic reactions often brings out parental questions about the relationship between physical and psychologic functioning. This is difficult for the pediatrician to deal with when a child's medical condition serves a neurotic purpose for one or both parents. Dealing with this problem requires tact and sensitivity. Simply telling parents that psychologic tests are in order because there is nothing medically wrong with such a child may result in angry, defensive and resistant reactions. Before mentioning psychologic evaluation, therefore, the pediatrician should endeavor to relate the physical complaints and symptoms to the child's feelings, worries, anxieties, etc. When so doing, he will begin to shift the focus of the parents' attention and concern to the child's inner suffering. Most parents are responsive to a child's emotional discomfort and will listen to suggestions pertaining to exploration of the situation.

Once a child is referred for psychologic testing, how the results will be handled becomes important. Informing the parents at the outset that the psychologist will discuss test data, impressions and implications directly with them removes the aura of secrecy which in the past has all too often become synonymous with psychologic tests. The pediatrician, however, should take care not to promise results which cannot be available from the tests (e.g., the source of the child's psychologic or learning problem). He should also tell them that he will be available to go over the implications of the data after the psychologist's final session with the family. Not only does this give parents an opportunity to ask questions at a time when their anxiety about the evaluation has abated, but it is comforting for them to know that someone with whom they are familiar is standing by to lend support to whatever difficult decision making and commitments are to be exercised following the evaluation.

Finally, as commonplace as referrals for psychologic evaluation are to pediatricians, these are still far from being viewed as a routine aspect of medical evaluation by parents and children. Consequently, one should never assume that the referral is accepted, understood, and passively responded to by parents and child. The manner in which the pediatrician handles the referral may have considerable bearing on whether it serves the purpose for which it was intended or whether the family perceives it as a frightening intrusion into their lives and that of their child. 